\title{
MiR-33a functions as a tumor suppressor in triple-negative breast cancer by targeting $\mathrm{EZH} 2$
}

\author{
Zeng Weihua, Zou Guorong, Cao Xiaolong and Li Weizhan * (1)
}

\begin{abstract}
Background: Increasing reports have confirmed that microRNAs play an important role in breast cancer progression, particularly in triple-negative breast cancer (TNBC). The aim of our study was to investigate the role of miR-33a in TNBC progression.

Methods: PCR assays were performed to detect miR-33a and EZH2 expression in TNBC tissues, adjacent nontumor tissues and cell lines. Western blot, CCK8, Transwell, cell colony formation and EdU cell proliferation, cell cycle analysis and luciferase reporter assays were used to determine the regulation of miR-33a/EZH2 in TNBC progression.

Results: MiR-33a was significantly downregulated in TNBC tissues and cell lines. MiR-33a overexpression in TNBC cells significantly inhibited cell growth and mobility and induced $\mathrm{G} 1$ cell cycle arrest. The luciferase reporter assay revealed that EZH2 is a direct target of miR-33a and that it was upregulated in TNBC tissues and cell lines. There was a negative correlation between miR-33a and EZH2 expression in TNBC tissues. EZH2 knockdown exerted similar inhibitory effects, while ectopic expression of EZH2 showed suppressive effects on malignant behaviors induced by miR-33a overexpression in TNBC cells.
\end{abstract}

Conclusions: These findings revealed that miR-33a is a tumor-suppressive miRNA in TNBC and can inhibit proliferation and mobility and induce G1 cell cycle arrest by directly targeting EZH2.

Keywords: TNBC, miR-33a, EZH2, Proliferation, Mobility

\section{Background}

Breast cancer (BC) is the most frequent and highly lethal tumor in women, and triple-negative breast cancer (TNBC) accounts for approximately $10-24 \%$ of BCs $[1,2]$. TNBC, the most aggressive type of $B C$ with a high proliferative and metastatic phenotype, is characterized by the absence of expression of the estrogen receptor $(\mathrm{ER})$, progesterone receptor $(\mathrm{PR})$ and human epidermal growth factor receptor 2 (HER-2) [1, 3]. TNBC has a worse prognosis, higher histological grade and displays

*Correspondence: 178038@qq.com

Department of Oncology, Panyu District Cancer Institute, Guangzhou Panyu Central Hospital, No. 8, Fuyu East Road, Qiaonan Street, Panyu

District, Guangzhou 511486, People's Republic of China high rates of drug resistance, metastasis and prost-surgical reoccurrence [4-7]. To date, there are still no efficient agents for the treatment of TNBC because of a lack of specific therapeutic targets $[8,9]$. Therefore, it is urgent to identify novel specific regulators in TNBC, which might provide new insights and clues for the development of more effective therapies for TNBC.

MicroRNAs (miRNAs) are a class of highly conserved small noncoding single-stranded RNA molecules [10]. They can regulate a variety of cellular processes, including cell proliferation, mobility, differentiation and metabolism, by base-pairing with the $3^{\prime}$-untranslated regions (3'-UTRs) of target genes [11]. An increasing number of studies have revealed that miRNA dysfunction contributes to $\mathrm{BC}$ development 
and metastasis [12, 13]. MiR-33a downregulation has been associated with cancer cell proliferation, mobility and chemotherapy sensitivity in various cancers, including lung cancer [14, 15], prostate cancer [16] and $B C[17,18]$, which suggested that it functions as a tumor suppressor. MiR-33a was upregulated after treatment with chidamide in TNBC and suppressed glycolysis by targeting LDHA [19]. It can also target ADAM9 and ROS1 to suppress BC proliferation and metastasis [18]. However, the detailed mechanisms of miR-33a in TNBC proliferation and mobility remain unclear.

The polycomb group protein enhancer of zeste homolog 2 (EZH2) is a histone-lysine $N$-methyltransferase enzyme that regulates DNA methylation and suppresses RNA transcription [20, 21]. EZH2 is highly expressed in many kinds of cancers including $B C$ [22], and EZH2 can regulate TNBC tumor growth and metastasis [23-25]. Knockdown of EZH2 suppressed TNBC MDA-MB-231 tumor growth and metastasis in xenograft models $[26,27]$. Tumor-suppressive miRNAs that directly target EZH2 to inhibit TNBC progression include miR-1301 [28] and miR-340 [25]. It has also been reported that EZH2 expression in TNBC patients is correlated with aggressiveness, advanced tumor stage and increased mortality [29, 30]. Therefore. EZH2 inhibition might be a promising potential therapeutic target for the treatment of TNBC. Whether EZH2 expression is associated with miR-33a levels in TNBC has not been determined.

In our study, we analyzed the expression of miR-33a and EZH2 in TNBC tissues and determined the role of miR-33a and EZH2 in TNBC progression. Our resulted uncovered an important role of EZH2 in the growth and mobility of TNBC cells and confirmed EZH2 was a target of miR-33a. Thus, targeting miR-33a/EZH2 signaling may be a potential strategy for TNBC treatment.

\section{Materials and methods Clinical specimens}

TNBC tissues $(n=60)$ and adjacent nontumor tissues $(\mathrm{n}=30)$, which included 15 TNBC tissues and 15 matched adjacent nontumor tissues from the same patients, were surgically obtained from Guangzhou Panyu Central Hospital. The tissues were stored in liquid nitrogen after collection, and all specimens were confirmed by pathological examination. Prior patient consent and approval from Guangzhou Panyu Central Hospital were obtained for the use of these clinical tissues in the study. This research was authorized by the Ethics Committees of Guangzhou Panyu Central Hospital (ethical number: NN-WK-2016115).

\section{Cells and cell culture}

The human triple-negative breast cancer cell lines MDAMB-231, MDA-MB-453, MDA-MB-468 and BT-549 and human normal breast epithelial cell line MCF10A were purchased from the Cell Bank of Type Culture Collection of Chinese Academy of Sciences (Shanghai, P.R. China). All the cells were cultured in recommendatory culture media containing $10 \% \mathrm{FBS}$ and $1 \%$ penicillin-streptomycin and maintained in a humidified atmosphere of $5 \%$ $\mathrm{CO}_{2}$ at $37^{\circ} \mathrm{C}$.

\section{Cell transfection}

For gene knockdown experiments, MDA-MB-231 and BT-594 cells were transfected with EZH2 siRNA (GenePharma) by Lipofectamine ${ }^{\text {TM }}$ RNAiMAX Transfection Reagent (Invitrogen) according to the protocol. For gene overexpression experiments, the cells were transfected with pCMV3-EZH2 plasmid, or miR-33a mimic by Lipofectamine $^{\mathrm{TM}} 3000$ Transfection Reagent (Invitrogen). After a 6-h transfection, medium containing transfection reagents were refreshed and cells were further cultured with fresh medium for $24 \mathrm{~h}$. The knockdown of EZH2 was performed with following siRNA duplex: 5'-GAG GGAAAGUGUAUGAUAATT- ${ }^{\prime}$ and $5^{\prime}$-UUAUCAUAC ACUUUCCCUCTT-3'.

\section{Quantitative real-time PCR}

Total RNA was extracted from TNBC tissues and cell lines using the E.Z.N.A. ${ }^{\circledR}$ Total RNA Kit I (Omega BioTek) according to the manufacturer's protocol. Reverse transcription was performed with the Transcriptor First Strand cDNA Synthesis Kit (Roche). Then, cDNA was amplified and quantified with the LightCycler 480 RealTime PCR System (Roche) using $2 \times$ SYBR Green I Master Mix (Bimake). For miRNA quantification, total RNA was reverse transcribed with the PrimeSript miRNA cDNA Synthesis Kit (TaKaRa), and the miR-33a cDNA was amplified and quantified with the LightCycler 480 Real-Rime PCR System (Roche) using $2 \times$ SYBR Green I Master Mix (Bimake). The levels of mRNA and miRNA were normalized to $A C T B$ and U6 levels, respectively. The $2^{-\Delta \Delta C T}$ method was used to determine relative gene expression.

\section{Western blot assay}

Total cell protein of TNBC cells was extracted by cell lysis in RIPA buffer (Thermo Fisher Scientific) containing protease and phosphatase inhibitor cocktails (Bimake). The protein concentrations were detected by a BCA Protein Assay Kit (Invitrogen). Proteins were separated by SDSPAGE, transferred onto polyvinylidene difluoride (PVDF) membranes (Millipore) and subjected to immunoblot 
analyses. The blot was performed with primary antibodies against EZH2 and GAPDH (1:1000 dilution. Cell Signaling Technology). The signals were detected by an HRP-conjugated secondary antibody (1:2000 dilution. Cell Signaling Technology) and the bands were visualized with an enhanced chemiluminescence (ECL, Millipore) system according to the manufacturer's protocol.

\section{Cell proliferation assay}

The effects of miR-33a and EZH2 on the proliferation of MDA-MB-231 and BT-549 cells were measured by the EdU cell proliferation assay (Beyotime Biotechnology) and CCK-8 assay (Beyotime Biotechnology). Briefly, cells were seeded in 96-well plates and cultured overnight. Cells were transfected with miRNA mimic, siRNA or plasmids for $6 \mathrm{~h}$, and the medium was replaced. For the EdU cell proliferation assay, cells were subjected to an EdU cell proliferation assay kit according to the standard protocol at $24 \mathrm{~h}$. The images were acquired with an inverted fluorescence microscope. For the CCK- 8 assay, the absorbance at $450 \mathrm{~nm}$ was measured using a microplate reader at $0,24,48,72$, and $96 \mathrm{~h}$.

\section{Colony formation assay}

Colony formation can be used to evaluate cell proliferation capacity. MDA-MB-231 and BT-549 cells were transfected with miRNA mimic, siRNA or plasmids for $24 \mathrm{~h}$, and then the cells were trypsinized and seeded into 6 -well plates at approximately 1000 cells per well. After culture for 10 days, the cells were fixed with $4 \%$ paraformaldehyde and stained with $0.1 \%$ crystal violet. The visible colonies of cells were observed and counted.

\section{Cell cycle analysis}

The effects of miR-33a and EZH2 on the cell cycle in MDA-MB-231 and BT-549 cells were detected using flow cytometry analysis. Cells were transfected with miRNA mimic, siRNA or plasmids for $48 \mathrm{~h}$, trypsinized, fixed with pre-cooled $70 \%$ ethanol and treated with $1 \mathrm{mg} / \mathrm{mL}$ RNase for $30 \mathrm{~min}$ at $37^{\circ} \mathrm{C}$. Then, the intracellular DNA was labeled with propidium iodide (PI) (Beyotime Biotechnology) for $30 \mathrm{~min}$ at $4{ }^{\circ} \mathrm{C}$ and analyzed by a flow cytometer (BD). The populations of TNBC cells in G1, S and G2/M phases were calculated with ModFit software (Verity Software House Inc., Topsham, ME, USA).

\section{Luciferase reporter assay}

The $3^{\prime}$-UTR of EZH2 containing miR-33a binding sites and its mutant were cloned into the pGL3-control luciferase reporter vector. The pGL3-EZH2 or mutant pGL3EZH2 plasmid was co-transfected with miR-33a or NC mimics into MDA-MB-231 and BT-549 cells. After a 48-h transfection, luciferase activity was evaluated by the
Dual-Luciferase Reporter Assay System (Promega) and was normalized to the activity of Renilla luciferase driven by a constitutively expressed promoter in the phRL vector.

\section{Cell migration and invasion assay}

The effects of miR-33a and EZH2 on the migration and invasion of MDA-MB-231 and BT-549 cells were measured by Transwell assays (Corning). For the Transwell migration assay, TNBC cells were first transfected with miRNA mimic, siRNA or plasmid. After transfection for $24 \mathrm{~h}$, cells were trypsinized, resuspended in serumfree medium and added into the upper filters. The lower chambers were filled with complete culture medium. After a 24-h incubation, the migrated cells were fixed with $4 \%$ paraformaldehyde and stained with $0.1 \%$ crystal violet. The cells on the inner sides of the upper filters were removed with cotton swabs, and the migrated cells on the bottom sides were imaged. For the invasion assay, the upper filters were precoated with diluted Matrigel (Corning), and the following procedures were the same as those for the Transwell migration assay.

\section{Statistical analysis}

Each experiment was repeated three times, and all statistical analyses were performed using GraphPad Prism 8.0 software. Data are presented as the mean \pm SEM. The differences between two groups were determined by two-tailed Student's $t$ test, and differences among more than two groups were analyzed using one-way ANOVA followed by the Tukey test. $P<0.05$ indicates a significant difference.

\section{Results}

\section{miR-33a is downregulated in TNBC tissues}

We first investigated the expression of miR-33a in TNBC tissues and adjacent nontumor tissues by qRT-PCR assay. As shown in Fig. 1a, the expression of miR-33a was significantly downregulated in TNBC tissues, compared to adjacent non-tumor tissues $(P<0.001)$. We also detected miR-33a expression in 15 pairs of TNBC tissues and matched adjacent nontumor tissues. Our results showed that miR-33a levels were much lower in TNBC tissues (Fig. 1b).

\section{Overexpression of miR-33a inhibits proliferation, mobility and induces $\mathrm{G} 1$ cell cycle arrest in TNBC cells}

Then, to further explore the effect of miR-33a on TNBC cell behaviors, miR-33a mimics were used. We first examined the expression of miR-33a in TNBC cell lines and qRT-PCR assay showed that miR-33a was significantly decreased in 4 TNBC cell lines (MDA-MB-231, MDA-MB-453, MDA-MB-468 and BT-549) than normal 

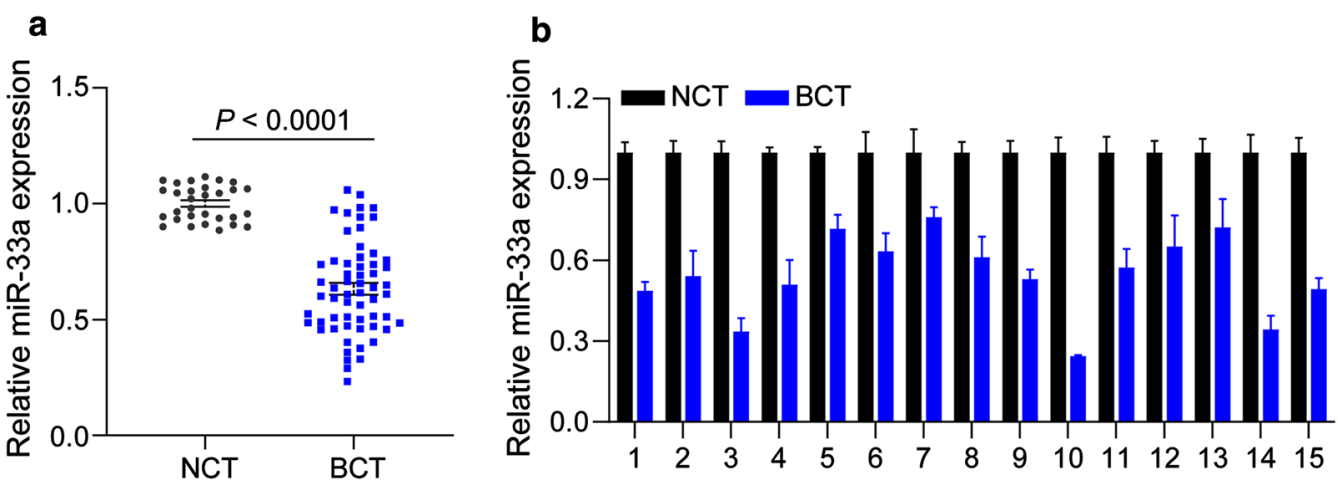

Fig. 1 miR-33a is downregulated in TNBC tissues. a The expression of miR-33a in 15 TNBC tissues and matched adjacent nontumor tissues was detected by PCR assay. $\mathbf{b}$ miR-33a expression was significantly lower in TNBC tissues $(n=75)$ than in adjacent nontumor tissues $(n=30)$. Data are shown as mean \pm SEM

MCF10A breast epithelial cells (Fig. 2a). MDA-231 and BT-549 cells were selected for further study due to the much lower miR-33a levels in these cells. We found that the expression of miR-33a increased by 4.5 - to 5.5 -fold in cells after transfection with miR-33a mimics (Fig. 2b). Then, cell proliferation was determined. We found that ectopic expression of miR-33a significantly decreased the number of EdU-positive cells (Fig. 2c, d). CCK-8 assay also showed that miR-33a suppressed the growth of MDA-MB-231 (Fig. 2e) and BT-549 (Fig. 2f) from 24 to $96 \mathrm{~h}$. In addition, miR-33a overexpression induced a 50\% reduction in colony formation capacities of TNBC cells (Fig. 2g). We also found that $C D K 4$, a cell proliferationassociated gene, was downregulated in TNBC cells after transfection with miR-33a mimics (Additional file 1: Figure S1A). Then, the effect of miR-33a on cell cycle was detected. Flow cytometric analysis showed that miR-33a mimics induced G1 cell cycle arrest in TNBC cells, but had less effect on the G2/M phase (Fig. 2h, i). Moreover, an in vitro Transwell assay was performed to test the effect of miR-33a on TNBC cells mobility. We found that the migrated and invasion capacities were reduced by nearly $50 \%$ in MDA-MB- 231 and BT- 549 cells transfected with miR-33a mimics (Fig. 2j-l). We also observed $M M P 9$ mRNA levels were significantly decreased in miR33a-trasfected cells, compared to cells transfected with NC mimics (Additional file 1: Figure S1B). Overall, miR33a inhibits proliferation and mobility and induces G1 cell cycle arrest in TNBC cells.

\section{EZH2 is a direct target of miR-33a}

Then, we searched for candidate target genes of miR33a using the databases, TargetScan, miRanda and PicTar (Fig. 3a). We found that a complementary miR33a sequence was present in the $3^{\prime}$-UTR of EZH2 mRNA (Fig. 3b), thus, EZH2 was selected for further investigations. Dual luciferase reporter assays showed that miR-33a overexpression clearly inhibited the activity of a luciferase reporter fused to the wild-type (WT) $3^{\prime}$-UTR of $E Z H 2$, but not of the mutant (MUT) reporter (Fig. 3c, d). In addition, the mRNA and protein levels of EZH2 in MDA-MB-231 and BT-549 cells were also determined. As expected, ectopic expression of miR-33a led to decreases more than 50\% in EZH2 mRNA (Fig. 3e) and protein (Fig. 3f, g) expressions. Thus, these results suggest that EZH2 is a direct target of miR-33a in TNBC cells.

\section{EZH2 is upregulated in TNBC tissues}

To corroborate the correlation between miR-33a and EZH2 in TNBC tissues, we further detected the expression of EZH2 in TNBC tissues and adjacent nontumor tissues. We found that EZH2 expression was remarkably higher in the TNBC tissues than the matched adjacent non-tumor tissues (Fig. 4a. $P<0.001$ ). QRT-PCR assay also revealed that the expression EZH2 in TNBC tissues was dramatically higher than adjacent nontumor tissues (Fig. 4b). Furthermore, a significantly negative correlation between miR-33a and EZH2 expression in TNBC tissues was observed (Fig. $4 \mathrm{c}, \mathrm{R}^{2}=0.4691, P=0.0048$ ). Collectively, the results demonstrate that EZH2 levels are upregulated and reveal a negative correlation with miR33a expression in TNBC tissues.

\section{EZH2 knockdown mimics the effects of miR-33a overexpression in TNBC cells}

Next, to verify that whether EZH2 is a key regulator of cellular behaviors in TNBC cells, EZH2 siRNA was used, which mimicked miR-33a overexpression in TNBC cells. We found that EZH2 expression was significantly increased in TNBC cell lines (MDA-MB-231, MDA-MB-453, MDA-MB-468 and BT-549) compared with normal breast epithelial cell line MCF10A 

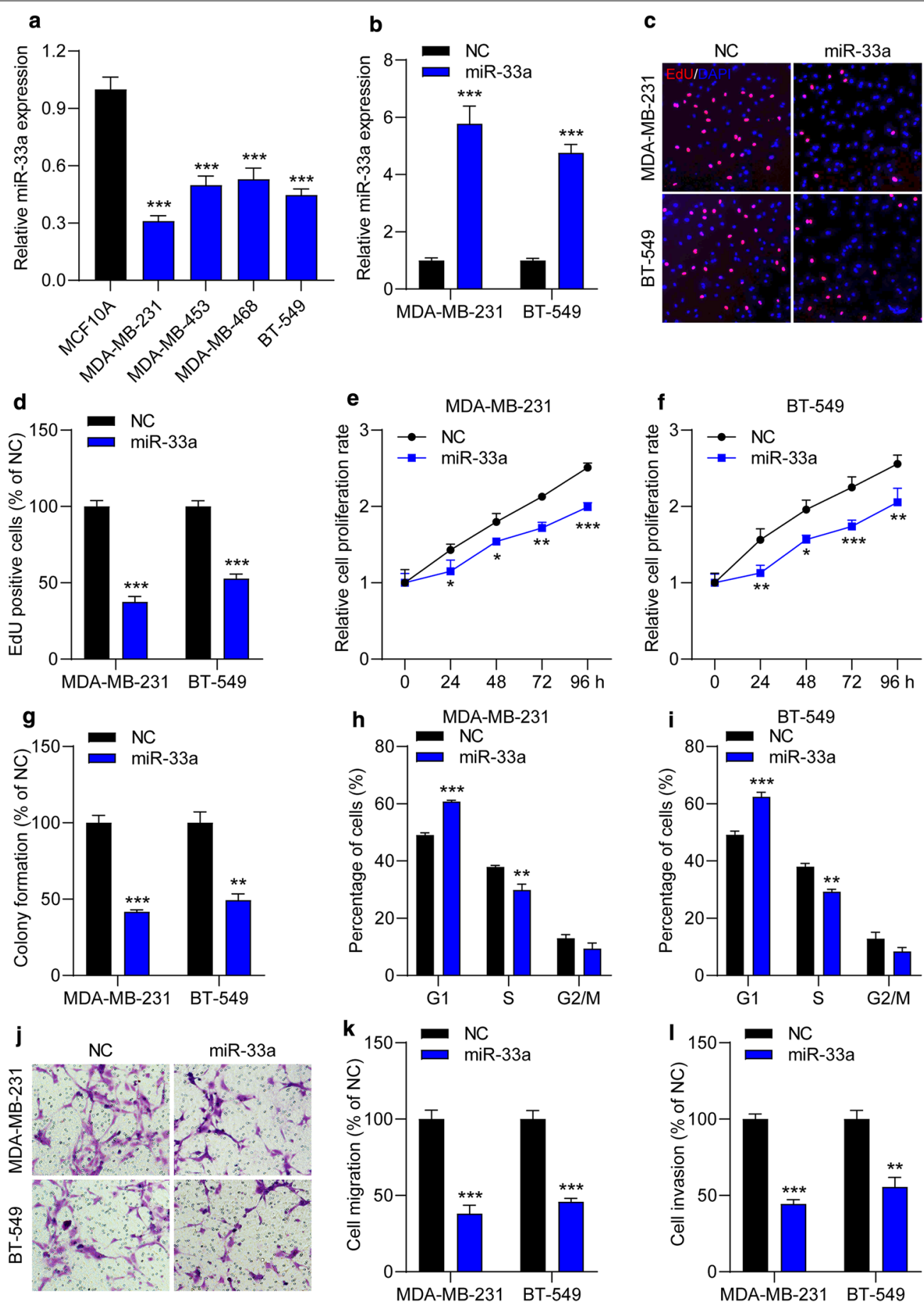

Fig. 2 Ectopic expression of miR-33a inhibits the malignant behaviors in TNBC cells. a The expression of miR-33a in TNBC cell lines and normal breast epithelial cells was measured by qRT-PCR assay. ${ }^{* *} P<0.001$ compared with MCF10A. $\mathbf{b}$ The expression of miR-33a in TNBC cell lines was elevated after transfection with miR-33a mimics. c, d MDA-MB-231 and BT-549 cells were transfected with NC or miR-33a mimics for $24 \mathrm{~h}$ and cell proliferation was determined by EdU cell proliferation kit. EdU positive cells and images were captured. $\mathbf{e}, \mathbf{f}$ The effect of miR-33a on cell proliferation rate was detected by CCK-8 assay. $\mathbf{g}$ Increased miR-33a reduced colony formation capacities in TNBC. $\mathbf{h}-\mathbf{I}$ TNBC cells were transfected with NC or miR-33a mimics for $48 \mathrm{~h}$ and then cell cycle analyses were performed by a flow cytometer. $\mathbf{j}, \mathbf{k}$ miR-33a mimics decreased the number of migrated $\mathbf{j}$, $\mathbf{k}$ and invasive (I) cells. Magnification, $\times 200$. Data are shown as mean \pm SEM $(n=3) .{ }^{*} P<0.05,{ }^{* *} P<0.01$ and ${ }^{* * *} P<0.001$ compared with cells transfected with NC mimics groups 


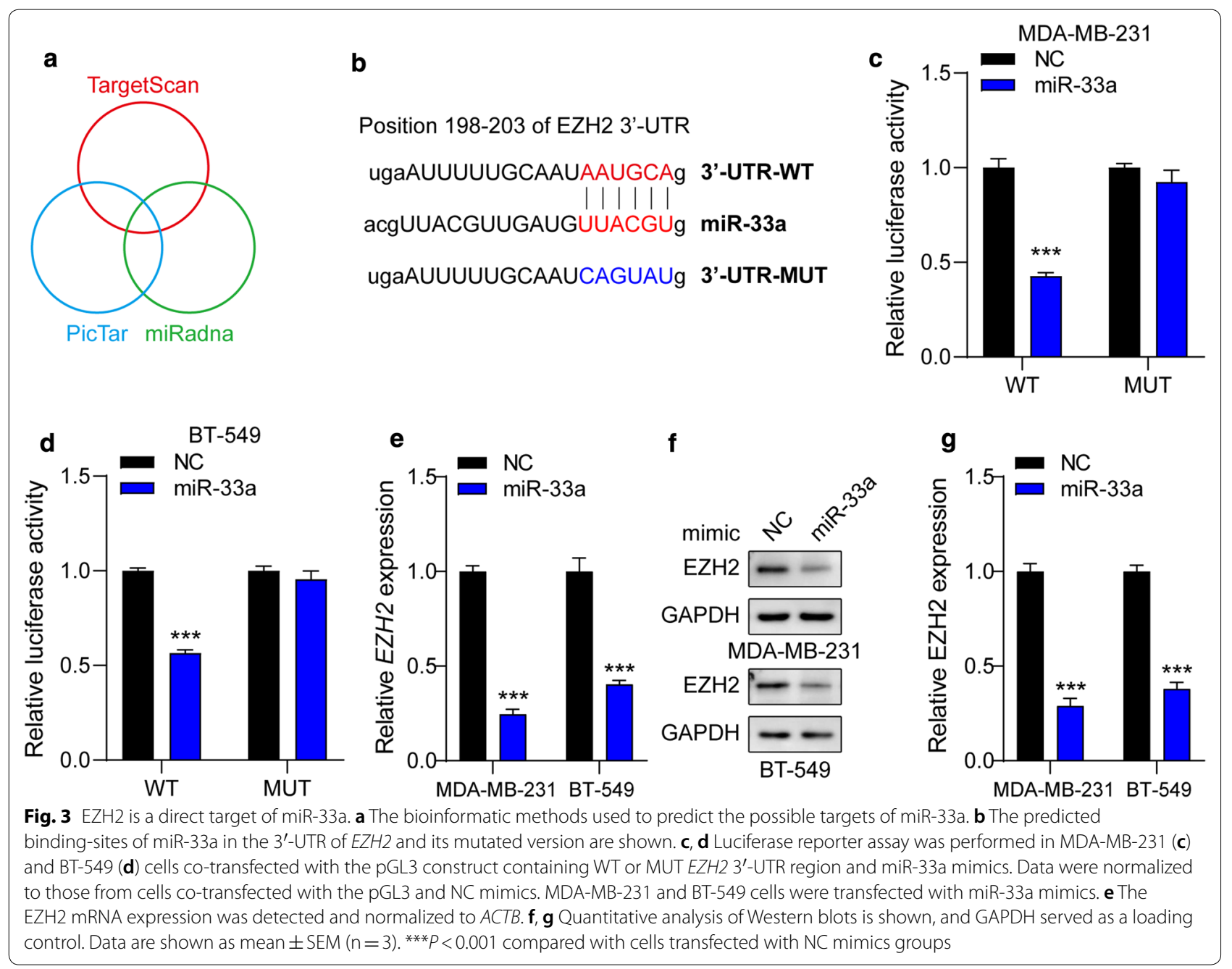

(Fig. 5a). Then, MDA-MB-231 and BT-549 cells were transfected with EZH2 siRNA to inhibit EZH2 expression (Fig. 5b, and Additional file 2: Figure S2A). We observed that the number of EdU-positive cells was reduced by $50 \%$ to $60 \%$ in cells transfected with EZH2 siRNA (Fig. 5c, d). The CCK-8 assay also showed that EZH2 siRNA dramatically inhibited TNBC cell growth from 24 to 96 h (Fig. 5e, f). Moreover, EZH2 inhibition significantly attenuated the colony formation capacity (Fig. 5g) and downregulated CDK4 mRNA levels (Additional file 2: Figure S2B) in both TNBC cell lines. Flow cytometric analysis revealed that $\mathrm{EZH} 2$ downregulation increased the percentage of TNBC cells arrested in G1 phase (Fig. 5h, i). Furthermore. the number of migrated (Fig. 5j, k) and invasive (Fig. 5l) cells was decreased $40 \%$ to $65 \%$ after transfection with EZH2 siRNA compared with NC siRNA. Moreover, the knockdown of EZH2 also downregulated the mRNA and protein levels of MMP9 expression in MDA-MB-231 and BT-549 cells (Additional file 2: Figure S2C). Taken together, EZH2 inhibition suppresses proliferation and mobility and induces $\mathrm{G} 1$ cell cycle arrest in TNBC cells.

\section{$\mathrm{EZH} 2$ is the key mediator of the effects of miR-33a in TNBC cells}

To further confirm that EZH2 is a direct target of miR33a, MDA-MB-231 and BT-549 were co-transfected with EZH2 overexpressing plasmid and miR-33a mimics. We first detected that transfection efficiency of the EZH2 overexpression plasmid in TNBC cells. As shown in Fig. 6a, b, the expression levels of EZH2 protein and mRNA were significantly upregulated in MDA-MB-231 and BT-549 cells after transfection with EZH2 overexpression plasmid, while can be attenuated by miR-33a mimics. Then, cell proliferation was first examined. We found that the number of EdU-positive cells in co-transfection groups was dramatically higher than cells transfected with miR-33a mimics (Fig. 6c-e). Compared to cells after transfection with miR-33a mimics, the proliferation rate is dramatically elevated in cells co-transfected 

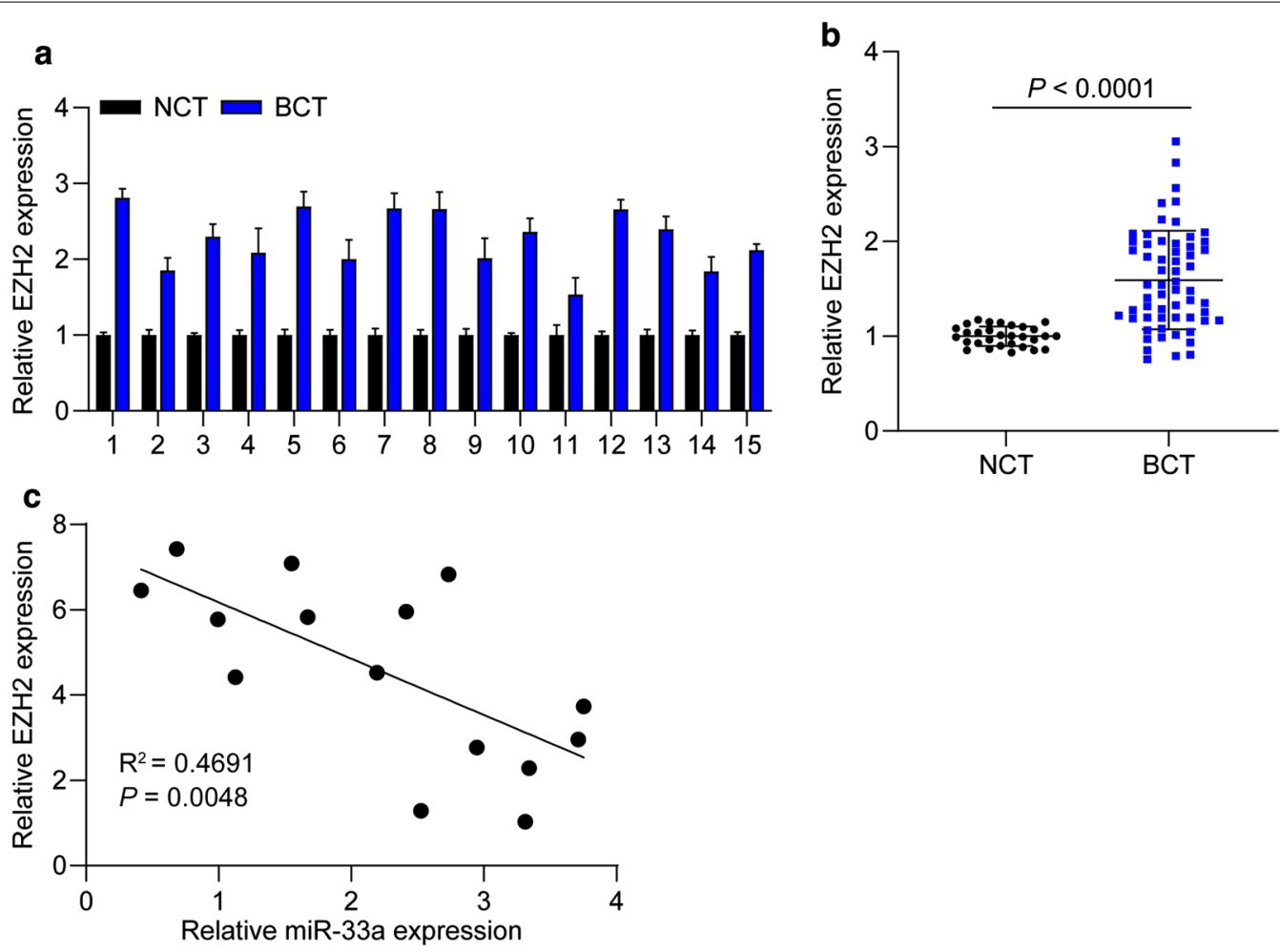

Fig. $4 \mathrm{EZH} 2$ is upregulated in TNBC tissues. a The expression of EZH2 in 15 TNBC tissues and matched adjacent nontumor tissues was determined using PCR assay. $\mathbf{b}$ EZH2 levels were significantly higher in TNBC tissues $(n=75)$ than in adjacent nontumor tissues $(n=30)$. $\mathbf{c}$ The correlation between miR-33a and EZH2 expression in TNBC tissues was analyzed. Data are shown as mean \pm SEM

with EZH2 overexpressing plasmid and miR-33a mimics (Fig. 6f, g). EZH2 overexpression also reversed the CDK4 mRNA decrease induced by miR-33a mimics (Additional file 3: Figure S3A). Furthermore, ectopic expression of EZH2 significantly decreased the number of TNBC cells arrested in G1 cell cycle (Fig. 6h, i). The number of migrated and invasive cells, and the expression levels of MMP9 mRNA and protein were significantly decreased in cells transfected with miR-33a mimics, which can be reversed by ectopic expression of EZH2 (Fig. 6j-l, and Additional file 3: Figure S3B). Together, these results demonstrate that EZH2 overexpression antagonizes miR-33a-mediated inhibitory effect on TNBC cell behaviors, which implies that EZH2 is a downstream target of miR-33a.

\section{Discussion}

Increased evidence supports that noncoding RNAs, including miRNAs, lncRNAs and circRNAs, are important regulators in tumor development, growth, metastasis and angiogenesis [31, 32]. MiRNAs can be oncogenic miRNAs and tumor-suppressive miRNAs [33, 34]. It was reported that miR-33a was downregulated in a variety of cancers, and ectopic expression of miR-33a suppressed multiple malignant behaviors [15-18]. miR33a can suppress epithelial-mesenchymal transition and metastasis in non-small cell lung cancer, and its expression in the blood of lung cancer patients was lower than that in healthy controls; thus, it could be regarded as a tumor suppressor and a novel biomarker for the diagnosis of lung cancer $[15,35]$. It also targets ST8SIA1 to suppress colorectal cancer progression [36] and targets HIF- $1 \alpha$ to inhibit melanoma cell growth and mobility [37]. Regarding breast cancer, decreased miR-33a in breast cancer tissues was associated with lymph node metastasis, and the expression of miR-33a was dramatically lower in metastatic breast cancer cell lines than in nonmetastatic cancer cell lines and normal breast epithelial cells [18]. Further study showed that miR-33a overexpression inhibited metastatic breast cancer cell growth and mobility in vitro and in vivo by targeting ADAM9 and ROS1 [18]. Therefore, miR-33a acts as a tumorsuppressive miRNA in most kinds of cancer. However, Wang $\mathrm{H}$ reported that upregulated expression of miR33a was correlated with poor prognosis of GBM patients and enhanced self-renewal of glioma-initiating cells via activation of the PKA and NOTCH pathways by targeting PDE8A and UVRAG [38]. Thus, miR-33a can act as 

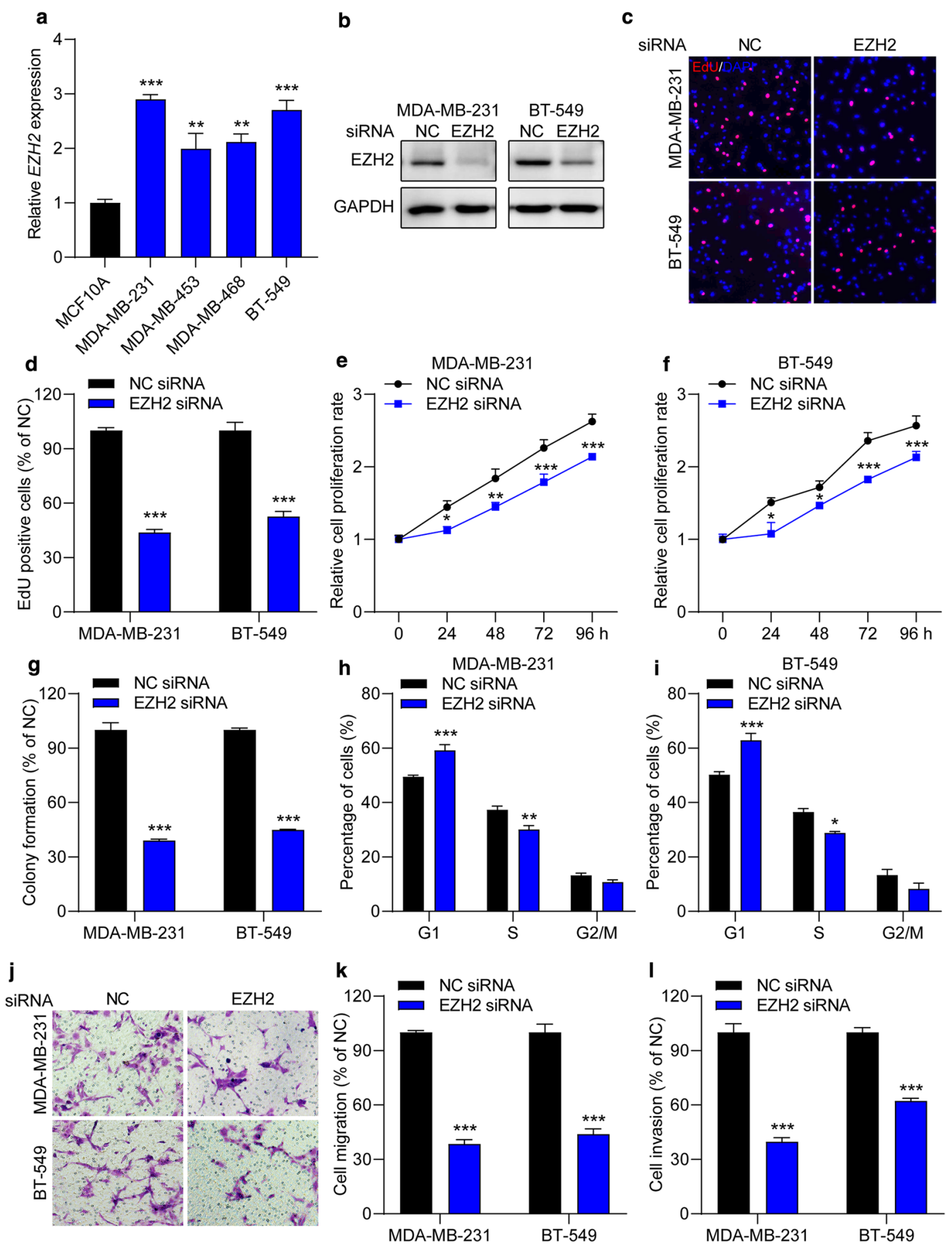

Fig. 5 The knockdown of EZH2 suppresses the malignant behaviors in TNBC cells. a The expression of EZH2 in TNBC cell lines and normal breast epithelial cell was measured by qRT-PCR assay. ${ }^{* *} P<0.01$, ${ }^{* *} P<0.001$ compared with MCF10A cells. $\mathbf{b}$ The expression of EZH2 in TNBC cell lines was significantly downregulated after transfection with EZH2 siRNA. c, d MDA-MB-231 and BT-549 cells were transfected with NC or EZH2 siRNA for $24 \mathrm{~h}$ and cell proliferation was determined by an EdU cell proliferation kit. EdU positive cells and images were captured. $\mathbf{e}, \mathbf{f} T$ The effect of EZH2 on cell proliferation rate was determined by CCK-8 assay. $\mathbf{g}$ Decreased miR-33a showed attenuated colony formation capacity in TNBC cells. h-ITNBC cells were transfected with NC or EZH2 siRNA for $48 \mathrm{~h}$ and then cell cycle analysis was performed by a flow cytometer. $\mathbf{j}, \mathbf{k}$ EZH2 siRNA decreased the number of migrated $(\mathbf{j}, \mathbf{k})$ and invasive (I) cells. Magnification, $\times 200$. Data are shown as mean $\pm \operatorname{SEM}(n=3) .{ }^{*} P<0.05,{ }^{* *} P<0.01$ and ${ }^{* * *} P<0.001$ compared with cells transfected with NC siRNA groups 

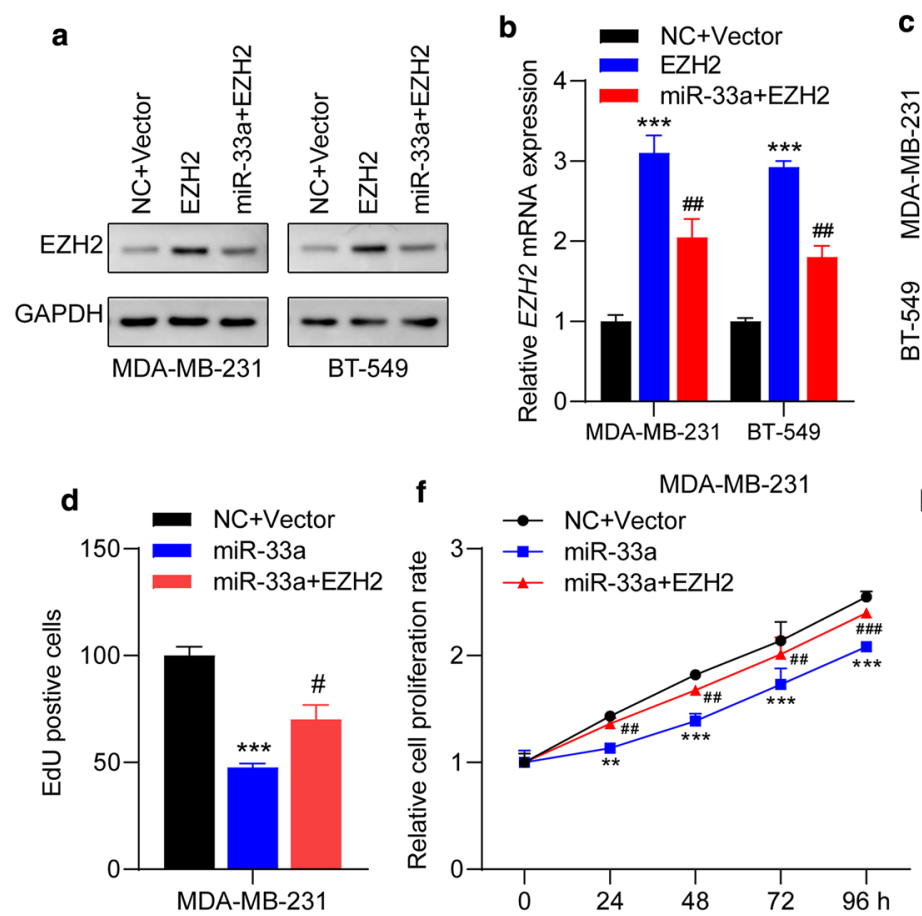

BT-549

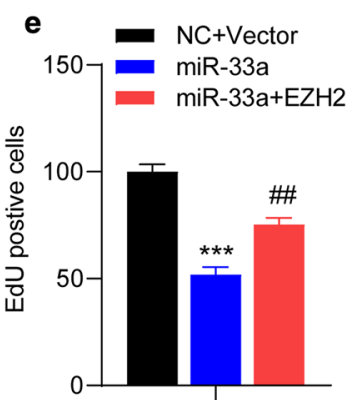

BT-549
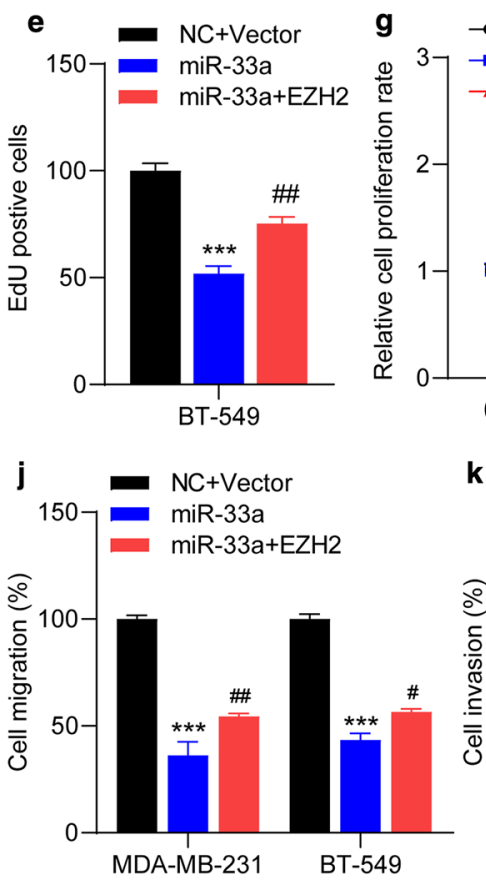

- NC+Vector

miR-33a

- miR-33a+EZH2
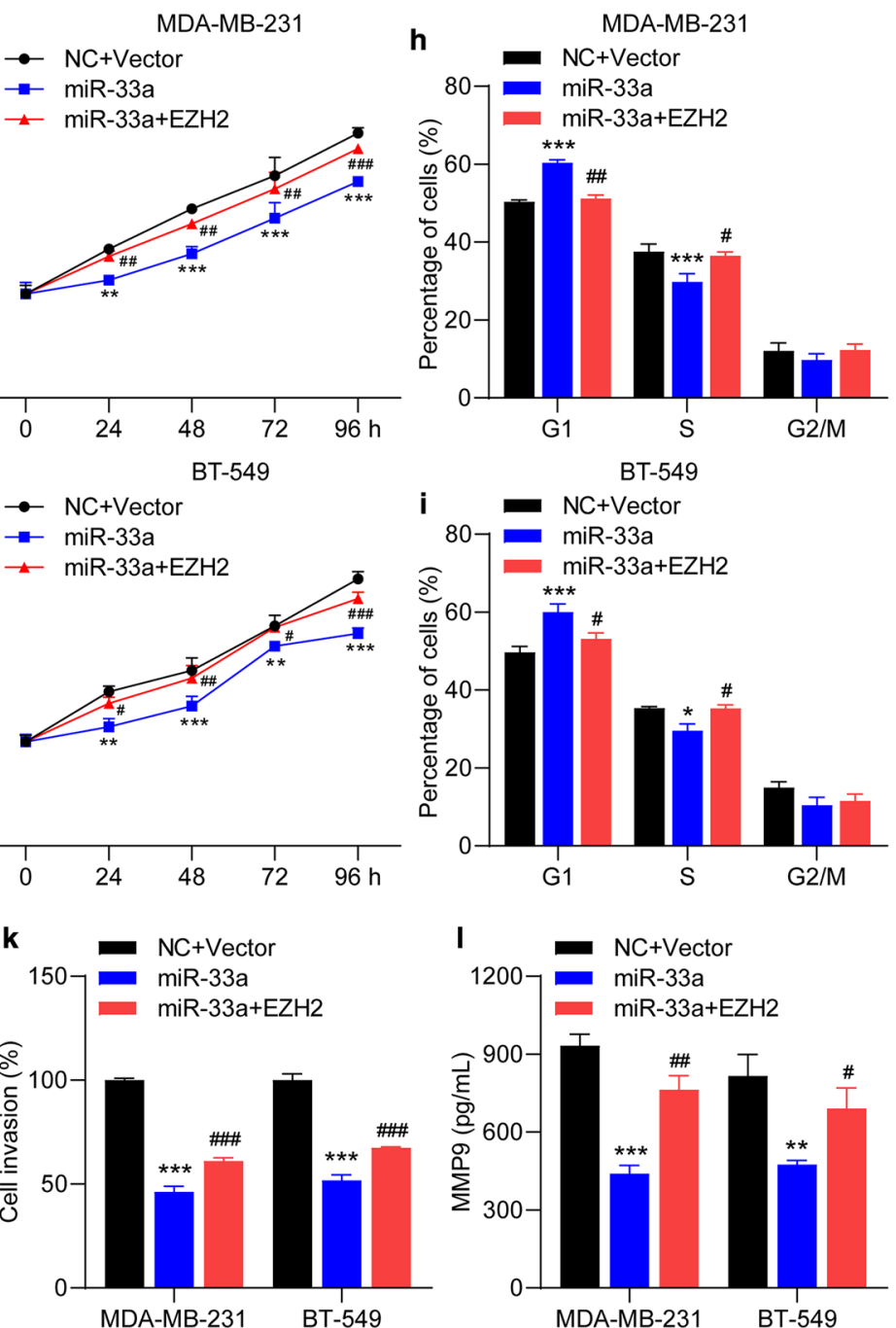

BT-549
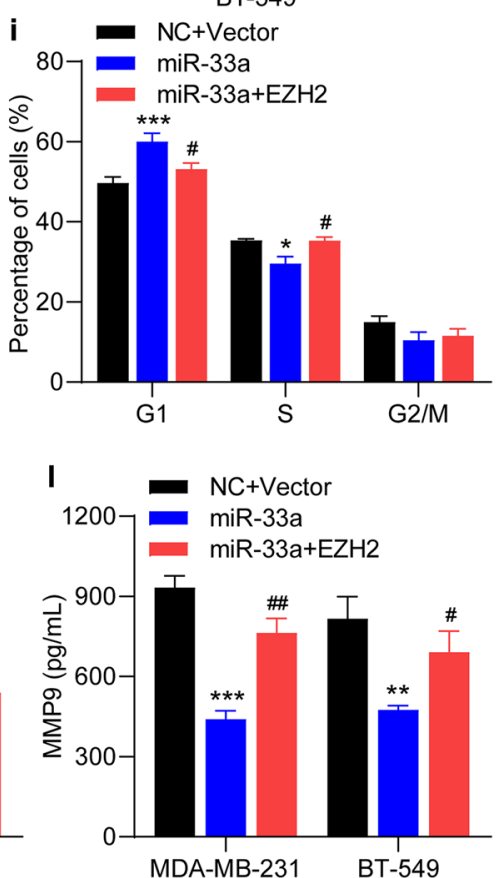

Fig. 6 miR-33a-mediated suppressive effects on malignant behaviors in TNBC cells can be offset by EZH2 overexpression. $\mathbf{a}$, b The expression of EZH2 in TNBC cells after indicated transfected was determined by Western blot and qRT-PCR assays. a Representative blots and $\mathbf{b}$ the levels of EZH2 mRNA after in cells indicated transfections are shown. c-e Ectopic expression of EZH2 counteracted miR-33a-mediated reduction of EdU incorporation in TNBC cells. Representative images (c) and the number of EdU-positive MDA-MB-231 (d) and BT-549 (e) cells are shown. f, $\mathbf{g}$ EZH2 overexpression promoted the proliferation rate in MDA-MB-231 and BT-549. $\mathbf{h}, \mathbf{i}$ Increased EZH2 reduced the percentage of cells arrested in G1 cell cycle mediated by miR-33a. j, k MiR-33a-induced decreases in cell migration and invasion capacities can be enhanced by ectopic expression of EZH2. I EZH2 overexpression promoted the secret of MMP9 in TNBC cells. Data are shown as mean \pm SEM $(n=3)$. ${ }^{*} P<0.05$, ${ }^{* *} P<0.01$ and ${ }^{* * *} P<0.001$ compared with cells transfected with $N C+$ Vector groups; ${ }^{\#} P<0.05,{ }^{\# \#} P<0.01$ and ${ }^{\# \#} P<0.001$ compared with cells transfected with miR-33a mimic groups 
either oncogenic or tumor-suppressive miRNA, which may depend on the tumor type. Nevertheless, the expression profiles and functions of miR-33a in triple-negative breast cancer growth and metastasis are less clear. In our study, we found that miR-33a was significantly downregulated in TNBC tissues compared with adjacent nontumor tissues, and further studies revealed that ectopic expression of miR-33a inhibited TNBC cell proliferation, colony formation, migration and invasion and induced G1 cell cycle arrest. miR-33a overexpression also downregulated TNBC cell CDK4 and MMP9 mRNA levels, which may account for the suppression of proliferation and mobility in TNBC cells.

EZH2, as a methyltransferase and component of PRC2, regulates H3K27 methylation to mediate gene transcriptional silencing [20]. EZH2 was higher in tumor tissues than in adjacent nontumor tissues and was associated with poor prognosis in tumor patients [39-41]. In breast cancer, its expression was correlated with breast cancer aggressiveness and could serve as an independent predictor of survival and recurrence [42]. A recent study reported that EZH2 promoted the mobility of TNBC cells by regulating the TIMP-2/MMP-2/9 pathway [23]. miRNAs were reported to regulate EZH2 expression in TNBC. Wu QJ reported that miR-1301 inhibited TNBC cell proliferation, migration and colony formation as well as xenograft growth by negatively regulating EZH2 expression [28]. Another study revealed that EZH2 reduction mediated by miR-340 mimic induced decreased expression of DNM1, H3K27me3, $\beta$-catenin and p-STAT3, which led to inhibition of miR-21 activity and upregulation of miR-200a/b, which contributed to inhibition of TNBC progression [25]. Here, our results showed that EZH2 expression was negatively correlated with miR-33a in TNBC tissues and was significantly higher in TNBC cell lines. Moreover, a luciferase reporter assay corroborated that miR-33a directly targeted EZH2 and inhibited EZH2 expression. EZH2 inhibition by EZH2 siRNA exerted similar effects on TNBC cell behaviors, while EZH2 upregulation by transfection with an EZH2-overexpressing plasmid reversed the suppressive effects mediated by miR-33a.

Many factors are responsible for the downregulation of tumor-suppressive miRNAs [43, 44]. It was reported that miR-33a downregulation in tumors may be associated with upregulation of proto-oncogenic lncRNAs, and this study showed that miR-33a inhibition mediated by IncRNA DANCR promoted osteosarcoma development and cancer stemness characteristics by inducing Axl upregulation [45]. EZH2 was also reported to induce silencing of tumor-suppressive miRNA I tumor cells, and miR-34a was epigenetically silenced by EZH2, which promoted cholangiocarcinoma cell growth by activating the Notch pathway [46]. Therefore, whether miR-33a downregulation in TNBC is mediated by $\mathrm{EZH} 2$ or oncogenic lncRNA remains to be further investigated.

Given that EZH2 was overexpressed in many types of cancer, and inhibitors targeting EZH2 were also developed. Tazemetostat, an EZH2 inhibitor, has been approved for treating epithelioid sarcoma, and it is the first EZH2 inhibitor approved by FDA [47]. Other EZH2 inhibitor, GSK343 and GSK236, were also reported to inhibit tumor progression in various cancer, such as glioblastoma [48], head and neck cancer, [49] and breast cancer [50]. Our in vitro study confirmed the regulatory mechanism of miR-33a/EZH2 cascade in TNBC progression, thus, more investigation of the effects of miR-33a and EZH2 on tumor growth and metastasis in vivo is needed. Moreover, EZH2 inhibitors can be used for in vitro and in vivo studies, which may further provide a rationale for potential therapeutic strategy for the treatment of TNBC patients.

\section{Conclusions}

In the current study, we verified an important role of miRNA-33a/EZH2 cascade in TNBC progression. We found that the expression of miR-33a was downregulated in TNBC tissues and cell lines, and it also had an inverse correlation with EZH2 expression in TNBC tissues. Our study confirmed that miR-33a was a tumor-suppressive miRNA in TNBC and indicated that EZH2 can be a potential therapeutic target for TNBC treatment.

\section{Supplementary information}

Supplementary information accompanies this paper at https://doi. org/10.1186/s12935-020-1160-z.

Additional file 1: Fig. 1 MiR-33a overexpression inhibited the levels of CDK4 and MMP9 mRNA expression in TNBC cells. TNBC cell were transfected with miR-33a mimics for $24 \mathrm{~h}$, and CDK4 (A) and MMP9 (B) mRNA levels were determined by qRT-PCR assay. Data are shown as mean \pm SEM $(\mathrm{n}=3) .{ }^{*} P<0.05,{ }^{* *} P<0.01$ and ${ }^{* *} P<0.001$ compared with cells transfected with NC mimics groups.

Additional file 2: Fig. 2 EZH2 siRNA downregulated the expression levels of EZH2, CDK4 and MMP9 in TNBC cells. (A) TNBC cells were transfected with different sets EZH2 siRNAs for $24 \mathrm{~h}$ and transfection efficiency was determined by qRT-PCR assay. (B-C) EZH2 siRNA downregulated the levels CDK4 mRNA (B), and MMP9 mRNA and protein (B) in TNBC cells. Data are shown as mean \pm SEM $(n=3) .{ }^{* *} P<0.001$ compared with cells transfected with NC mimics groups

Additional file 3: Fig. 3 Ectopic expression of EZH2 attenuated miR33a-induced downregulation of CDK4 and MMP9 expression. (A-B) TNBC cells were co-transfected with miR-33a and EZH2 overexpressing plasmid for $24 \mathrm{~h}$, and the expression of CDK4 (A) and MMP9 (B) mRNA were measured by qRT-PCR assay. Data are shown as mean $\pm \operatorname{SEM}(n=3)$. ${ }^{* * *} P<0.001$ compared with cells transfected with NC+Vector groups; ${ }^{\# \#} P<0.01$ and ${ }^{\# \#} P<0.001$ compared with cells transfected with miR-33a mimics groups. 


\section{Abbreviations}

3'-UTR: 3'-Untranslated regions; BC: Breast cancer; CCK8: Cell counting kit-8; EdU: 5-Ethynyl-2-deoxyuridine; ER: Estrogen receptor; EZH2: Enhancer of zeste homolog-2; HER-2: Human epidermal growth factor receptor 2; PI: Propidium iodide; PR: Progesterone receptor; PVDF: Polyvinylidene difluoride; SDS-PAGE: Polyacrylamide gel electrophoresis; TNBC: Triple negative breast cancer.

\section{Acknowledgements}

None.

\section{Authors' contributions}

WZ and WL conceived and designed this study. GZ and XC collected the samples and performed the experiment. WZ wrote the paper. WL revised the manuscript. All authors read and approved the final manuscript.

\section{Funding}

None.

\section{Availability of data and materials}

The analyzed data sets generated during the study are available from the corresponding author on reasonable request.

\section{Ethics approval and consent to participate}

The manuscript contains data of human tissue procured from Guangzhou Panyu Central Hospital, after internal ethical approval and prior patient consent for publication. The samples were processed at Guangzhou Panyu Central Hospital for experimental purposes. The proposed experimental plan and procedures have been reviewed and approved by the Ethics Committee of the the Guangzhou Panyu Central Hospital (ethical number: NN-WK-2016115). All experiments were performed strictly in accordance with relevant guidelines and regulations.

\section{Consent for publication}

All authors have read the manuscript and provided their consent for publication.

\section{Competing interests}

The authors declare that they have no competing interests.

Received: 15 December 2019 Accepted: 29 February 2020 Published online: 18 March 2020

\section{References}

1. Carey L, Winer E, Viale G, Cameron D, Gianni L. Triple-negative breast cancer: disease entity or title of convenience? Nat Rev Clin Oncol. 2010;7(12):683-92.

2. Yeo SK, Guan JL. Breast cancer: multiple subtypes within a tumor? Trends Cancer. 2017;3(11):753-60.

3. Dent R, Trudeau M, Pritchard Kl, Hanna WM, Kahn HK, Sawka CA, Lickley LA, Rawlinson E, Sun P, Narod SA. Triple-negative breast cancer: clinical features and patterns of recurrence. Clin Cancer Res. 2007;13(15 Pt 1):4429-34

4. Sorlie T. Molecular portraits of breast cancer: tumour subtypes as distinct disease entities. Eur J Cancer. 2004;40(18):2667-75.

5. Weigelt B, Hu Z, He X, Livasy C, Carey LA, Ewend MG, Glas AM, Perou CM, Van't Veer $L$. Molecular portraits and 70-gene prognosis signature are preserved throughout the metastatic process of breast cancer. Cancer Res. 2005;65(20):9155-8.

6. Liedtke C, Mazouni C, Hess KR, Andre F, Tordai A, Mejia JA, Symmans WF, Gonzalez-Angulo AM, Hennessy B, Green M, et al. Response to neoadjuvant therapy and long-term survival in patients with triple-negative breast cancer. J Clin Oncol. 2008;26(8):1275-81.

7. Badve S, Dabbs DJ, Schnitt SJ, Baehner FL, Decker T, Eusebi V, Fox SB, Ichihara S, Jacquemier J, Lakhani SR, et al. Basal-like and triple-negative breast cancers: a critical review with an emphasis on the implications for pathologists and oncologists. Mod Pathol. 2011;24(2):157-67.
8. Lehmann BD, Pietenpol JA Identification and use of biomarkers in treatment strategies for triple-negative breast cancer subtypes. J Pathol. 2014:232(2):142-50.

9. Szekely B, Silber AL, Pusztai L. New therapeutic strategies for triple-negative breast cancer. Oncology (Williston Park). 2017;31(2):130-7.

10. He L, Hannon GJ. MicroRNAs: small RNAs with a big role in gene regulation. Nat Rev Genet. 2004:5(7):522-31.

11. Bartel DP. MicroRNAs: target recognition and regulatory functions. Cell. 2009:136(2):215-33.

12. Serpico D, Molino L, Di Cosimo S. microRNAs in breast cancer development and treatment. Cancer Treat Rev. 2014;40(5):595-604.

13. Khoshnaw SM, Green AR, Powe DG, Ellis IO. MicroRNA involvement in the pathogenesis and management of breast cancer. J Clin Pathol. 2009;62(5):422-8.

14. Du M, Zhang Y, Mao Y, Mou J, Zhao J, Xue Q, Wang D, Huang J, Gao S, Gao Y. MiR-33a suppresses proliferation of NSCLC cells via targeting METTL3 mRNA. Biochem Biophys Res Commun. 2017;482(4):582-9.

15. Pan J, Zhou C, Zhao X, He J, Tian H, Shen W, Han Y, Chen J, Fang S, Meng $X$, et al. A two-miRNA signature (miR-33a-5p and miR-128-3p) in whole blood as potential biomarker for early diagnosis of lung cancer. Sci Rep. 2018:8(1):16699.

16. Karatas OF, Wang J, Shao L, Ozen M, Zhang Y, Creighton CJ, Ittmann M. miR-33a is a tumor suppressor microRNA that is decreased in prostate cancer. Oncotarget. 2017:8(36):60243-56.

17. Wolfe AR, Bambhroliya A, Reddy JP, Debeb BG, Huo L, Larson R, Li L, Ueno NT, Woodward WA. MiR-33a decreases high-density lipoproteininduced radiation sensitivity in breast cancer. Int J Radiat Oncol Biol Phys. 2016;95(2):791-9.

18. Zhang C, Zhang Y, Ding W, Lin Y, Huang Z, Luo Q. MiR-33a suppresses breast cancer cell proliferation and metastasis by targeting ADAM9 and ROS1. Protein Cell. 2015:6(12):881-9.

19. Bai X, Jiang H, Han G, He Q. Chidamide suppresses the glycolysis of triple negative breast cancer cells partially by targeting the miR33a5pLDHA axis. Mol Med Rep. 2019;20(2):1857-65.

20. Vire E, Brenner C, Deplus R, Blanchon L, Fraga M, Didelot C, Morey L, Van Eynde A, Bernard D, Vanderwinden JM, et al. The Polycomb group protein EZH2 directly controls DNA methylation. Nature. 2006;439(7078):871-4.

21. Bachmann IM, Halvorsen OJ, Collett K, Stefansson IM, Straume O, Haukaas SA, Salvesen HB, Otte AP, Akslen LA. EZH2 expression is associated with high proliferation rate and aggressive tumor subgroups in cutaneous melanoma and cancers of the endometrium, prostate, and breast. J Clin Oncol. 2006:24(2):268-73.

22. Kleer CG, Cao Q, Varambally S, Shen R, Ota I, Tomlins SA, Ghosh D, Sewalt RG, Otte AP, Hayes DF, et al. EZH2 is a marker of aggressive breast cancer and promotes neoplastic transformation of breast epithelial cells. Proc Natl Acad Sci USA. 2003;100(20):11606-11.

23. Chien YC, Liu LC, Ye HY, Wu JY, YU YL. EZH2 promotes migration and invasion of triple-negative breast cancer cells via regulating TIMP2-MMP-2/-9 pathway. Am J Cancer Res. 2018;8(3):422-34.

24. Hartman ZC, Poage GM, den Hollander P, Tsimelzon A, Hill J, Panupinthu N, Zhang Y, Mazumdar A, Hilsenbeck SG, Mills GB, et al. Growth of triple-negative breast cancer cells relies upon coordinate autocrine expression of the proinflammatory cytokines IL-6 and IL-8. Cancer Res. 2013;73(11):3470-80

25. Shi Z, Li Y, Qian X, Hu Y, Liu J, Zhang S, Zhang J. MiR-340 inhibits triplenegative breast cancer progression by reversing EZH2 mediated miRNAs dysregulated expressions. J Cancer. 2017;8(15):3037-48.

26. Gonzalez ME, Li X, Toy K, DuPrie M, Ventura AC, Banerjee M, Ljungman $M$, Merajver SD, Kleer CG. Downregulation of EZH2 decreases growth of estrogen receptor-negative invasive breast carcinoma and requires BRCA1. Oncogene. 2009;28(6):843-53.

27. Moore HM, Gonzalez ME, Toy KA, Cimino-Mathews A, Argani P, Kleer CG. EZH2 inhibition decreases p38 signaling and suppresses breast cancer motility and metastasis. Breast Cancer Res Treat. 2013;138(3):741-52.

28. Wu Q, Chen Z, Zhang G, Zhou W, Peng Y, Liu R, Chen C, Feng J. EZH2 induces the expression of miR-1301 as a negative feedback control mechanism in triple negative breast cancer. Acta Biochim Biophys Sin (Shanghai). 2018;50(7):693-700.

29. De Brot M, Rocha RM, Soares FA, Gobbi H. Prognostic impact of the cancer stem cell related markers $\mathrm{ALDH} 1$ and $\mathrm{EZH} 2$ in triple negative and basal-like breast cancers. Pathology. 2012;44(4):303-12. 
30. Hussein YR, Sood AK, Bandyopadhyay S, Albashiti B, Semaan A, Nahleh Z, Roh J, Han HD, Lopez-Berestein G, Ali-Fehmi R. Clinical and biological relevance of enhancer of zeste homolog 2 in triple-negative breast cancer. Hum Pathol. 2012;43(10):1638-44.

31. Anastasiadou E, Jacob LS, Slack FJ. Non-coding RNA networks in cancer. Nat Rev Cancer. 2018;18(1):5-18.

32. Nicolas FE. Role of ncRNAs in development, diagnosis and treatment of human cancer. Recent Pat Anticancer Drug Discov. 2017;12(2):128-35.

33. Zhang B, Pan X, Cobb GP, Anderson TA. microRNAs as oncogenes and tumor suppressors. Dev Biol. 2007;302(1):1-12.

34. Kent OA, Mendell JT. A small piece in the cancer puzzle: microRNAs as tumor suppressors and oncogenes. Oncogene. 2006;25(46):6188-96.

35. Yang L, Yang J, Li J, Shen X, Le Y, Zhou C, Wang S, Zhang S, Xu D, Gong Z. MircoRNA-33a inhibits epithelial-to-mesenchymal transition and metastasis and could be a prognostic marker in non-small cell lung cancer. Sci Rep. 2015;5:13677.

36. Shan Y, Liu Y, Zhao L, Liu B, LiY, Jia L. MicroRNA-33a and let-7e inhibit human colorectal cancer progression by targeting ST8SIA1. Int J Biochem Cell Biol. 2017;90:48-58.

37. Zhou J, Xu D, Xie H, Tang J, Liu R, Li J, Wang S, Chen X, Su J, Zhou X, et al. miR-33a functions as a tumor suppressor in melanoma by targeting HIF1alpha. Cancer Biol Ther. 2015;16(6):846-55.

38. Wang H, Sun T, Hu J, Zhang R, Rao Y, Wang S, Chen R, McLendon RE, Friedman AH, Keir ST, et al. miR-33a promotes glioma-initiating cell self-renewal via PKA and NOTCH pathways. J Clin Invest. 2014;124(10):4489-502.

39. Morera L, Lubbert $M$, Jung $M$. Targeting histone methyltransferases and demethylases in clinical trials for cancer therapy. Clin Epigenetics. 2016;8:57.

40. Simon JA, Lange CA. Roles of the EZH2 histone methyltransferase in cancer epigenetics. Mutat Res. 2008:647(1-2):21-9.

41. Yan KS, Lin CY, Liao TW, Peng CM, Lee SC, Liu YJ, Chan WP, Chou RH. EZH2 in cancer progression and potential application in cancer therapy: a friend or foe? Int J Mol Sci. 2017;18:6.

42. Collett K, Eide GE, Arnes J, Stefansson IM, Eide J, Braaten A, Aas T, Otte AP, Akslen LA. Expression of enhancer of zeste homologue 2 is significantly associated with increased tumor cell proliferation and is a marker of aggressive breast cancer. Clin Cancer Res. 2006;12(4):1168-74.

43. Cohen A, Burgos-Aceves MA, Smith Y. microRNAs downregulation in cancer is associated with guanine enrichment in the terminal loop sequences of their precursors. Microrna. 2018;7(1):20-7.

44. Williams M, Cheng YY, Blenkiron C, Reid G. Exploring mechanisms of MicroRNA downregulation in cancer. Microrna. 2017:6(1):2-16.

45. Jiang N, Wang X, Xie X, Liao Y, Liu N, Liu J, Miao N, Shen J, Peng T. IncRNA DANCR promotes tumor progression and cancer stemness features in osteosarcoma by upregulating AXL via miR-33a-5p inhibition. Cancer Lett. 2017:405:46-55.

46. Kwon H, Song K, Han C, Zhang J, Lu L, Chen W, Wu T. Epigenetic silencing of miRNA-34a in human cholangiocarcinoma via EZH2 and DNA methylation: impact on regulation of notch pathway. Am J Pathol. 2017;187(10):2288-99.

47. First EZH2 inhibitor approved-for rare sarcoma. Cancer discovery. 2020.

48. Stazi G, Taglieri L, Nicolai A, Romanelli A, Fioravanti R, Morrone S, Sabatino M, Ragno R, Taurone S, Nebbioso M, Carletti R. Dissecting the role of novel EZH2 inhibitors in primary glioblastoma cell cultures: effects on proliferation, epithelial-mesenchymal transition, migration, and on the pro-inflammatory phenotype. Clin Epigenet. 2019;11(1):1-7.

49. Zhou L, Mudianto T, Ma X, Riley R, Uppaluri R. Targeting EZH2 Enhances Antigen Presentation, Antitumor Immunity, and Circumvents Anti-PD-1 Resistance in Head and Neck Cancer. Clin Cancer Res. 2020;26(1):290-300,

50. Puppe J, Opdam M, Schouten PC, Jóźwiak K, Lips E, Severson T, van de Ven M, Brambillasca C, Bouwman P, van Tellingen O, Bernards R. EZH2 is overexpressed in BRCA1-like breast tumors and predictive for sensitivity to high-dose platinum-based chemotherapy. Clin Cancer Res. 2019;25(14):4351-62.

\section{Publisher's Note}

Springer Nature remains neutral with regard to jurisdictional claims in published maps and institutional affiliations.

Ready to submit your research? Choose BMC and benefit from:

- fast, convenient online submission

- thorough peer review by experienced researchers in your field

- rapid publication on acceptance

- support for research data, including large and complex data types

- gold Open Access which fosters wider collaboration and increased citations

- maximum visibility for your research: over $100 \mathrm{M}$ website views per year

At BMC, research is always in progress.

Learn more biomedcentral.com/submissions 